\title{
Molecular characteristics of diffuse large B-cell lymphoma in the Positron Emission Tomography-Guided Therapy of Aggressive Non-Hodgkin lymphomas (PETAL) trial: correlation with interim PET and outcome
}

\author{
Julia Richter (1)', Andreas Hüttmann $\mathbb{E}^{2}$, Jan Rekowski $\mathbb{B}^{3}$, Christine Schmitz ${ }^{2}$, Selina Gärtner ${ }^{4}$, Andreas Rosenwald ${ }^{5}$, \\ Martin-Leo Hansmann ${ }^{6}$, Sylvia Hartmann $\mathbb{0}^{6}$, Peter Möller ${ }^{7}$, Hans-Heinrich Wacker ${ }^{8}$, Alfred Feller ${ }^{9}$, Christoph Thorns ${ }^{10}$, \\ Stefan Müller ${ }^{11}$, Ulrich Dührsen ${ }^{2}$ and Wolfram Klapper (iD)
}

Dear Editor,

Treatment results in diffuse large B-cell lymphoma (DLBCL) are heterogeneous. Established risk models, like the International Prognostic Index (IPI) or molecular features such as MYC translocations and the cell of origin (COO) subtype, are associated with outcome ${ }^{1}$. In the Positron Emission Tomography-Guided Therapy of Aggressive Non-Hodgkin Lymphomas (PETAL) trial, interim positron emission tomography (iPET) after two cycles of rituximab, cyclophosphamide, doxorubicin, vincristine, and prednisone (R-CHOP) has recently been shown to predict outcome independently of the IPI ${ }^{2}$. Whether molecular high-risk features of aggressive B-cell lymphomas are correlated with (and may predict) an unfavorable (positive) iPET result, has not been studied in detail. We aimed to understand the molecular features of DLBCL with a positive iPET by investigating known molecular risk groups, such as $M Y C, B C L 2$, and $B C L 6$ translocations, and subgroups defined by the $\mathrm{COO}$

Correspondence: Wolfram Klapper (wklapper@path.uni-kiel.de)

${ }^{1}$ Department of Hematopathology, University Hospital Schleswig-Holstein, Kiel, Germany

${ }^{2}$ Department of Hematology, University Hospital Essen, University of DuisburgEssen, Essen, Germany

Full list of author information is available at the end of the article.

These authors contributed equally: Julia Richter, Andreas Hüttmann

These authors jointly supervised this work: Ulrich Dührsen, Wolfram Klapper concept which may benefit from targeted therapies (e.g., activated B-cell-like [ABC] lymphomas).

In the PETAL trial, patients with a positive iPET scan after two cycles of R-CHOP were randomized to receive six additional cycles of R-CHOP or six blocks of an intensive Burkitt's lymphoma protocol ${ }^{2}$. Patients with a negative scan were continued on R-CHOP. Scans were evaluated using the $\triangle S U V \max$ method $^{3}$. A positive interim PET was defined by a decrease of SUVmax at interim PET by $\leq 66 \%$ compared with baseline. A decrease $>66 \%$ was considered a negative finding ${ }^{2}$.

Available formalin-fixed paraffin-embedded specimens were analyzed for $\mathrm{COO}$ by gene expression using the HTG EdgeSeq System (HTG Molecular Diagnostics, Tucson, AZ, USA). MYC and BCL2 and/or BCL6 translocations were assessed by fluorescence in situ hybridization (FISH, Vysis-Abbott, Des Plaines, IL, USA). Survival curves for event-free survival (EFS) and overall survival (OS) were compared using hazard ratios (HR) with 95\% confidence intervals (CI) from Cox regression and the log-rank test. In addition, we performed multivariable Cox regression analyses for EFS and OS that included $\mathrm{COO}$ by gene expression, $M Y C$ break, "doublehit" status, IPI risk groups (low-risk group includes the IPI risk groups low and low-intermediate, and high-risk group includes the groups high intermediate and high), 
Table 1 Molecular features of iPET-negative and iPET-positive DLBCL

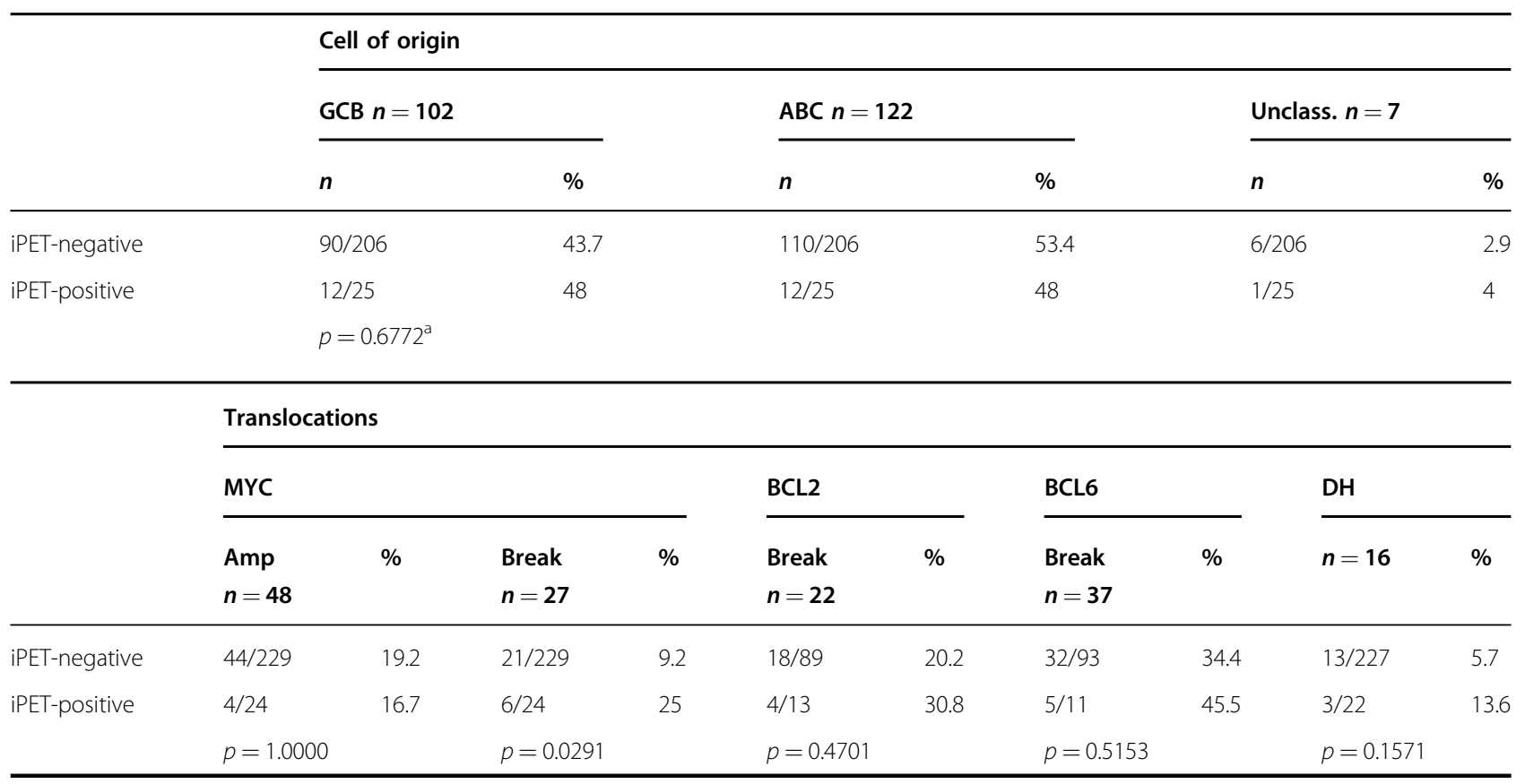

GE gene expression, GCB germinal center like, $A B C$ activated B-cell like, unclass. unclassified, FISH fluorescence in situ hybridization, $A m p$ amplification, $D H$ double-hit, $n$ indicates number of positive/number of all cases with data

${ }^{a}$ Fisher's exact test $\mathrm{GCB}$ versus non-GCB

and $\mathrm{PPET}$ result. COO by gene expression profiling was available for 239 patients eight of whom failed the quality control, leaving 231 specimens with gene expression results (Table 1). FISH data were obtained from 253 lymphomas. In 196 cases, FISH for $M Y C$ and gene expression data were available.

Of 609 DLBCL belonging to the intention-to-treat population of the PETAL trial, 546 (89.7\%) were iPETnegative and $63(10.3 \%)$ were iPET-positive ${ }^{2}$. First, we investigated the effect of the molecular parameters on outcome (OS; EFS) in the whole DLBCL cohort, i.e., irrespective of iPET result. Concordance of $\mathrm{COO}$ assessment indicated by agreement in classification between immunohistochemistry according to Hans et al. ${ }^{4}$ and gene expression was $83.8 \%$. COO as assessed by gene expression profiling showed no statistical significant association with outcome ( $p=0.2077$ for EFS, $p=0.2020$ for OS for GCB subtype; Fig. 1a, b). BCL2 breaks were not associated with outcome (data not shown). By contrast, BCL6 breaks were associated with decreased survival time (HR 2.105, 95\% CI 1.067-4.153, $p=0.0282$ for EFS; HR 2.783, 95\% CI 1.010-7.671, $p=0.0388$ for OS). An association of $B C L 6$ breaks with survival has only been shown in one previous study ${ }^{5}$. Recent data suggest that BCL6 translocations are enriched in the DLBCL category of unclassified $\mathrm{COO}$. They are often associated with other genetic aberrations, such as $N O T C H$ mutations ${ }^{6,7}$. In contrast to our observation, the subgroups enriched for BCL6 translocations published so far were characterized by superior survival ${ }^{6}$. A more comprehensive analysis of the mutational landscape of the lymphomas included in the PETAL trial may help resolve this discrepancy.

$M Y C$ breaks showed a trend for inferior EFS (HR 1.601, 95\% CI $0.879-2.915, p=0.1206)$ and statistically significantly reduced OS (HR 2.531, 95\% CI 1.240-5.166, $p=0.0083$; Fig. 1c, d). A "double-hit" status (MYC translocation and $B C L 2$ or $B C L 6$ translocation in the same lymphoma specimen) had previously been shown to be associated with unfavorable outcome ${ }^{8}$. In line with these findings, we observed that "double-hit" was associated with inferior EFS (HR 2.036, 95\% CI 1.019-4.068, $p=0.0400$ ) and OS (HR 3.006, 95\% CI 1.343-6.726, $p=$ 0.0049; Fig. 1e, f). In multivariable analysis, only IPI highrisk group compared with low-risk group and positive iPET retained a statistically significant association with EFS (HR 3.828, 95\% CI 1.664-8.809, $p=0.0016$ for IPI; HR 3.326, 95\% CI 1.544-7.163, $p=0.0021$ for iPET) and OS (HR 5.076, 95\% CI 1.558-16.532, $p=0.0070$ for IPI; HR 3.447, 95\% CI 1.293-9.190, $p=0.0134$ for iPET).

In a second step, we assessed the relationship between molecular features and iPET results. The proportion of GCB cases as determined by gene expression profiling was similar in the iPET-negative and iPET-positive groups (90/206, $43.7 \%$ and $12 / 25,48.0 \%$, respectively, $p=0.6772$; Table 1). However, lymphomas with a positive iPET scan were significantly enriched for $M Y C$ translocations (6/25, 


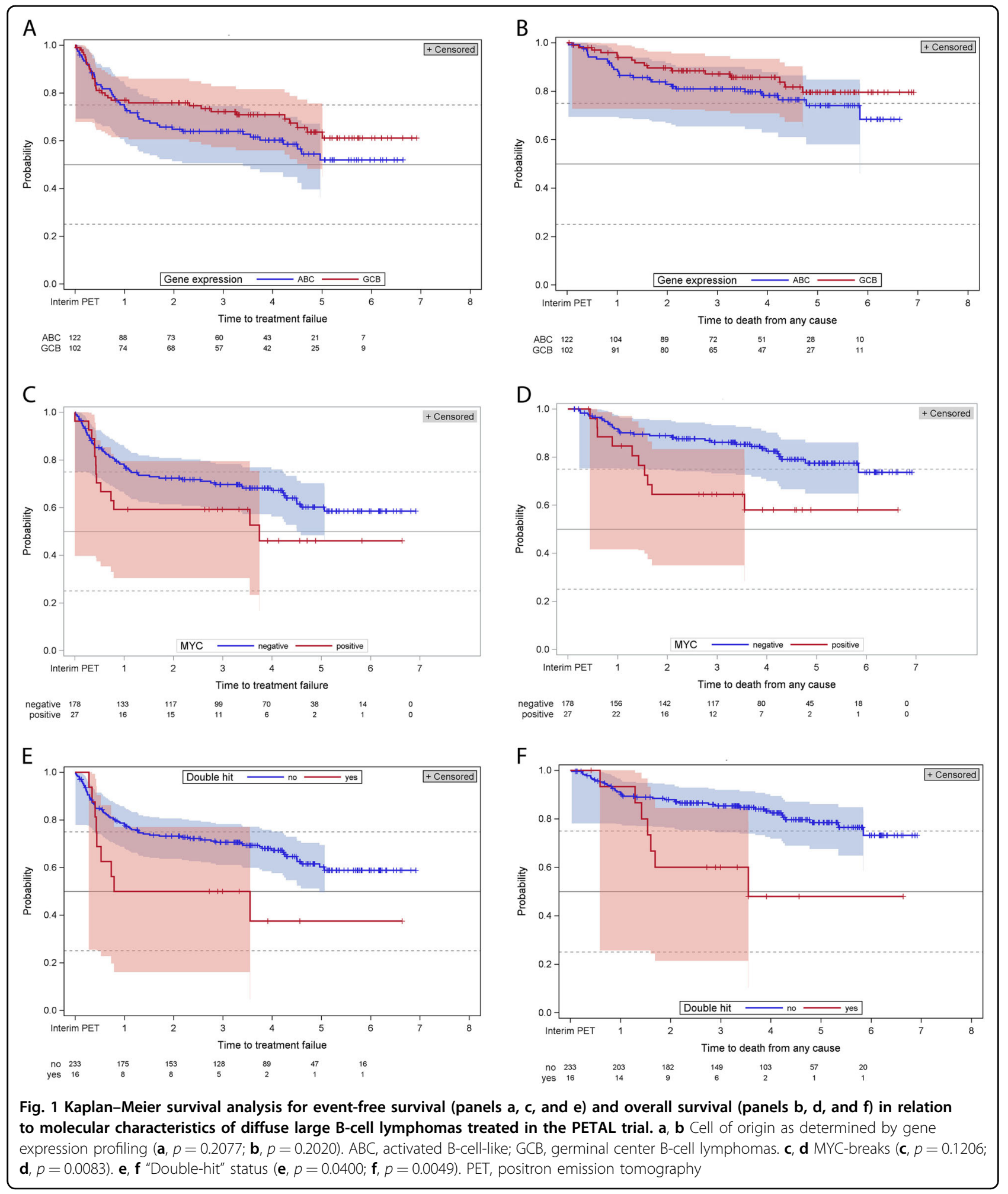

24.0\%) as compared with iPET-negative lymphomas (21/ $241,8.7 \%, p=0.0394$; Table 1). We did not detect a statistically significant difference between iPET-positive and iPET-negative lymphomas with respect to $M Y C$ amplifications, BCL2 or BCL6 breaks, or "double-hit" status (Table 1).

In a subgroup of 510 DLBCL patients participating in the PETAL trial, we recently confirmed the prognostic 
impact of baseline total metabolic tumor volume on outcome. Using the $41 \%$ maximum standardized uptake value method for measuring tumor volume, the best threshold to distinguish between patients with good versus poor outcome was $328 \mathrm{~cm}^{3}$ (Schmitz et al., submitted). Neither COO nor MYC, BCL2, BCL6 or "double-hit" translocations were associated with tumor volume (data not shown).

In DLBCL, PET scanning provides prognostic information independent of molecular features such as $\mathrm{COO}$ or expression of BCL2 and MYC protein ${ }^{9}$. In one small study, a positive iPET was significantly associated with $M Y C$ translocations ${ }^{10}$. To the best of our knowledge, our study represents the first comprehensive analysis of $\mathrm{COO}$ and translocation status in a large prospective trial investigating the value of iPET under controlled conditions. In our study, $\mathrm{COO}$ was not associated with outcome as observed in other large prospective trials ${ }^{5,8,11}$. In contrast, lymphomas with MYC translocations with or without additional BCL2 or BCL6 breaks were found to be associated with inferior EFS and OS, confirming the prognostic relevance of this biomarker. In the revised version of the World Health Organization classification of tumors of hematopoietic and lymphoid tissues, which was published after completion of the PETAL trial, "doublehit" lymphomas are separated from DLBCL and classified as high grade B-cell lymphomas with MYC and BCL2 or BCL6 translocations ${ }^{1}$. We confirmed their inferior prognosis in the present investigation. However, the subgroup of "double-hit" lymphomas randomized to receive the Burkitt's lymphoma protocol was too small $(n=3)$ to investigate the impact of treatment intensification on outcome. Regarding the whole group of iPET-positive patients, intensification of therapy did not improve survival $^{2}$.

In summary, MYC breaks with or without "double-hit" status were significantly associated with a positive iPET scan. Yet, the unfavorable prognosis of a positive iPET cannot solely be explained by MYC or "double-hit" translocations because most iPET-positive lymphomas lacked these genetic abnormalities. Our results strengthen the role of iPET as a prognostic tool, independent not only of IPI, but also of COO and MYC translocation status. Intensification of conventional chemotherapy failed to improve survival in iPET-positive lymphomas ${ }^{2}$. A more comprehensive molecular characterization of this subgroup may allow us to identify molecular pathways amenable to targeted treatment approaches.

\section{Acknowledgements}

Funded by Deutsche Krebshilfe (grants no. 107592 and 110515), Amgen Germany, and Roche Pharma. Infrastructure support by the KKI (KinderKrebshilfe Buchholz Holm-Seppensen) is gratefully acknowledged.

\section{Author details}

${ }^{1}$ Department of Hematopathology, University Hospital Schleswig-Holstein, Kiel, Germany. ${ }^{2}$ Department of Hematology, University Hospital Essen, University of Duisburg-Essen, Essen, Germany. Institute for Medical Informatics, Biometry and Epidemiology, University Hospital Essen, University of Duisburg-Essen, Essen, Germany. ${ }^{4}$ HTG Molecular Diagnostics Inc, Tucson, AZ 85706, USA. ${ }^{5}$ Department of Pathology, University of Würzburg, Würzburg, Germany. ${ }^{6}$ Department of Pathology, University Hospital Frankfurt, Frankfurt, Germany. ${ }^{7}$ Department of Pathology, University Hospital Ulm, Ulm, Germany. ${ }^{8}$ Institut für Hämatopathologie, Kiel, Germany. ${ }^{9}$ Hämatopathologie Lübeck, Lübeck, Germany. ${ }^{10}$ Department of Pathology, University Hospital Schleswig-Holstein, Lübeck, Germany. ${ }^{11}$ Department of Nuclear Medicine, University Hospital Essen, University of Duisburg-Essen, Essen, Germany

\section{Conflict of interest}

The authors declare the following disclosures: Richter-HTG Molecular Diagnostics, Inc.: research funding. Hüttmann - Celgene: travel expenses; Roche: travel expenses. Gärtner-HTG Molecular Diagnostics, Inc.: employment. Dührsen—Amgen: research funding; Celgene: honoraria, research funding; Roche: honoraria, research funding; AbbVie: consultancy, honoraria; Gilead: consultancy, honoraria; Janssen: honoraria. Klapper-HTG Molecular Diagnostics, Inc.: research funding; Amgen: honoraria, research funding; Regeneron: honoraria, research funding; Hoffman-La Roche: honoraria, research funding; Takeda: honoraria, research funding.

\section{Publisher's note}

Springer Nature remains neutral with regard to jurisdictional claims in published maps and institutional affiliations.

Received: 8 April 2019 Revised: 15 May 2019 Accepted: 22 May 2019 Published online: 19 August 2019

\section{References}

1. Swerdlow, S. H. et al. WHO Classification of Tumors of Haematopoietic and Lymphoid Tissues, revised 4th edn. (International Agency for Reaserch on Cancer, 2018).

2. Duhrsen, U. et al. Positron emission tomography-guided therapy of aggressive non-Hodgkin lymphomas (PETAL): a multicenter, randomized phase III trial. J. Clin. Oncol. 36, 2024-2034 (2018).

3. Lin, C. et al. Early 18F-FDG PET for prediction of prognosis in patients with diffuse large B-cell lymphoma: SUV-based assessment versus visual analysis. J. Nucl. Med. 48, 1626-1632 (2007).

4. Hans, C. P. et al. Confirmation of the molecular classification of diffuse large B-cell lymphoma by immunohistochemistry using a tissue microarray. Blood 103, 275-282 (2004).

5. Horn, H. et al. Different biological risk factors in young poor-prognosis and elderly patients with diffuse large B-cell lymphoma. Leukemia 29, 1564-1570 (2015).

6. Schmitz, R. et al. Genetics and pathogenesis of diffuse large B-cell lymphoma. N. Engl. J. Med. 378, 1396-1407 (2018).

7. Chapuy, B. et al. Molecular subtypes of diffuse large B cell lymphoma are associated with distinct pathogenic mechanisms and outcomes. Nat. Med. 24 679-690 (2018).

8. Copie-Bergman, C. et al. MYC-IG rearrangements are negative predictors of survival in DLBCL patients treated with immunochemotherapy: a GELALYYSA study. Blood 126, 2466-2474 (2015).

9. Cottereau, A. S. et al. Molecular profile and FDG-PET/CT total metabolic tumor volume improve risk classification at diagnosis for patients with diffuse large B-cell lymphoma. Clin. Cancer Res. 22, 3801-3809 (2016).

10. Yuan, L. et al. Prognostic analysis of interim (18)F-FDG PET/CT in patients with diffuse large $B$ cell lymphoma after one cycle versus two cycles of chemotherapy. Eur. J. Nucl. Med. Mol. Imaging 46, 478-488 (2019).

11. Cunningham, D. et al. Rituximab plus cyclophosphamide, doxorubicin, vincristine, and prednisolone in patients with newly diagnosed diffuse large B-cell non-Hodgkin lymphoma: a phase 3 comparison of dose intensification with 14-day versus 21-day cycles. Lancet. 381, 1817-1826 (2013). 